\title{
\begin{tabular}{l|l} 
Mibraries & DSpace@MIT
\end{tabular}
}

\author{
MIT Open Access Articles
}

\section{Multimaterial Piezoelectric Fibres}

The MIT Faculty has made this article openly available. Please share how this access benefits you. Your story matters.

Citation: Egusa, S. et al. “Multimaterial Piezoelectric Fibres." Nature Materials 9.8 (2010): 643648. CrossRef. Web.

As Published: http://dx.doi.org/10.1038/nmat2792

Publisher: Nature Publishing Group

Persistent URL: http://hdl.handle.net/1721.1/78317

Version: Author's final manuscript: final author's manuscript post peer review, without publisher's formatting or copy editing

Terms of Use: Article is made available in accordance with the publisher's policy and may be subject to US copyright law. Please refer to the publisher's site for terms of use. 


\section{Multimaterial Piezoelectric Fibres}

S. Egusa, ${ }^{1}$ Z. Wang, ${ }^{1,2}$ N. Chocat, ${ }^{3}$ Z. M. Ruff, ${ }^{3}$ A. M. Stolyarov, ${ }^{1,4}$ D. Shemuly, ${ }^{3}$ F. Sorin, ${ }^{1,3}$ P. T. Rakich, ${ }^{1,5}$ J. D. Joannopoulos, ${ }^{1,2}$ Y. Fink ${ }^{1,3^{*}}$

${ }^{1}$ Research Laboratory of Electronics, ${ }^{2}$ Department of Physics, and ${ }^{3}$ Department of Materials Science and Engineering, Massachusetts Institute of Technology, 77 Massachusetts Avenue, Cambridge, Massachusetts 02139

${ }^{4}$ School of Engineering and Applied Sciences, Harvard University, 29 Oxford Street, Cambridge, Massachusetts 02138

${ }^{5}$ Present Address: Sandia National Laboratories, P.O. Box 5800, Albuquerque, New Mexico 87185

* To whom correspondence should be addressed. E-mail: yoel@mit.edu 
Fibres have evolved over eons from simple textile yarns to complex fibre-optic communication systems. Nevertheless, a key premise has remained unchanged: fibres function as static devices or sensors, incapable of controllably changing properties at arbitrary frequencies. Enabling electrically-modulated fibres could pave the way for unprecedented technological capabilities. Previous approaches have focused on refractive index modulation ${ }^{1-4}$, non-linear optical mechanisms realized in silica glass fibres ${ }^{5-8}$, and electro-actively modulated polymer fibres ${ }^{9}$. These implementations are inherently limited by simple geometries, short fibre lengths and high driving fields. Here we lift these limitations through the composition of an internal phase that is simultaneously crystalline and noncentrosymmetric. Tens of metres of fibres containing a thin ferroelectric polymer layer spatially confined and electrically contacted by internal viscous electrodes are thermally drawn entirely from a macroscopic preform. The viscous state of the draw enables fibre cross sections of variable symmetry and resulting acoustic wave fronts. Heterodyne optical and acoustic measurements substantiate the piezoelectric response in fibres of $\mathrm{C}_{2}$ and $\mathrm{C}_{\infty}$ symmetries from kilohertz to megahertz. A singlefibre electrically-driven device containing a high-Q Fabry-Perot optical resonator and a piezoelectric transducer is fabricated and measured.

A promising path for introducing rapid modulation into fibres would be through the piezoelectric effect ${ }^{10-11}$. Embedding piezoelectric domains would allow fibres to be electrically actuated over broad frequencies on the one hand, and to function as sensitive broadband microphones on the other. However, fibres for the most part have been made of materials in the disordered glassy state precluding the crystalline symmetry requirements necessary for piezoelectricity.

Recent progress in drawing of fibres made of a multiplicity of materials ${ }^{12}$ present new opportunities for re-examining this challenge. With this approach, fibre materials are drawn from 
preforms in a regime dominated by viscous forces allowing for internal low viscosity domains to be arranged in non-equilibrium cross sections confined by viscous glassy boundary layers. In fact constructing a piezoelectric fibre could be accomplished in a straightforward manner by assembling a preform made of a piezoelectric material poly(vinylidene fluoride) (PVDF) ${ }^{13-14}$, with metal electrodes and an insulating polymer, which would be followed by a thermal draw. The stress present during the fibre draw should in principle induce the non-polar $\alpha$ to the ferroelectric $\beta$ phase transition in the PVDF layer ${ }^{13,15-16}$. The process should yield many metres of fibre with built-in internal electrodes which could be utilized to establish the large electric field necessary for poling the PVDF layer. However, upon detailed examination a number of significant challenges and seemingly conflicting requirements arise. The necessity to utilize crystalline materials both for the piezoelectric layer and the electrical conductors leads to the formation of multiple adjacent low viscosity and high aspect ratio domains. These domains undergoing a reduction in cross sectional dimensions are susceptible to capillary breakup and mixing during fibre drawing due to flow instabilities. Layer thickness non-uniformity either in the lateral or in the longitudinal directions ${ }^{17-18}$ precludes the formation of the coercive field needed for poling. Moreover, even if capillary breakup were kinetically averted and uniform sections of fibres were to emerge they would not exhibit piezoelectricity because the stress and strain conditions necessary to induce the thermodynamic phase transition in PVDF cannot be sustained in the fibre draw process.

To address these challenges we choose to focus our attention on the ability to maintain geometric coherence and layer thickness uniformity. A viscous and conductive carbon-loaded poly(carbonate) (CPC) is used to confine the low viscosity crystalline piezoelectric layer during 
the draw process. The CPC layers exhibit high viscosity $\left(10^{5} \sim 10^{6} \mathrm{~Pa} \cdot \mathrm{s}\right)$ at the draw temperature and adequate conductivity $\left(1 \sim 10^{4} \mathrm{ohm} \cdot \mathrm{m}\right)$ over the frequency range from $\mathrm{DC}$ to tens of $\mathrm{MHz}$, thus facilitating short range (hundreds of microns) charge transport on length scales associated with the fibre cross section. Then a piezoelectric polymer which crystallizes into the appropriate phase is identified. Poly(vinylidene-fluoride-Trifluoroethylene) copolymer $(\mathrm{P}(\mathrm{VDF}-\mathrm{TrFE}))^{19}$ assumes the ferroelectric $\beta$ phase spontaneously upon solidification from the melt ${ }^{20-21}$ without necessitating any mechanical stress, making it particularly suitable for the thermal fibre drawing process.

The constituent fibre materials are then assembled as illustrated in Figure 1a. A series of shells comprising a $700 \mu \mathrm{m}$-thick layer of $\mathrm{P}(\mathrm{VDF}-\mathrm{TrFE})$ (70:30 molar ratio, Solvey; meltpressed from pellets) and $250 \mu \mathrm{m}$-thick layers of CPC are assembled with indium filaments and a poly(carbonate) (PC) cladding. The entire structure is consolidated at $210^{\circ} \mathrm{C}$ to remove trapped gas and form high quality interfaces. The preform is then thermally drawn in a furnace at $230^{\circ} \mathrm{C}$ into fibres more than 100-metre long. Scanning electron microscopy (SEM) images of the fibre cross section show the $\mathrm{P}(\mathrm{VDF}-\mathrm{TrFE})$ layer (40 $\mu \mathrm{m}$ thick) sandwiched between CPC layers, with the shape and the aspect ratio unchanged from those of the preform (Figure 1b). Wide angle Xray diffraction $(\mathrm{XRD})$ measurements of $\mathrm{P}(\mathrm{VDF}-\mathrm{TrFE})$ copolymer domains harvested from the drawn fibres, are compared to the material used for the preforms. Both specimen exhibit identical peaks at $2 \theta=19.9,35.2$, and 40.7 degrees, which correspond to $(200) /(110),(001)$ and (310)/(020), (111)/(201) and (400)/(220) peaks of the $\beta$ phase $^{22}$ respectively (Figure $\left.1 \mathrm{c}\right)$, thus establishing that the drawn copolymer solidifies in its $\beta$ phase. The crystallinity fraction as calculated from XRD patterns is over $90 \%$. The obtained fibre is then poled by applying through 
the internal fibre electrodes an electric field in excess of $60 \mathrm{MV} / \mathrm{m}$ which is above the literature value $^{23}$ of coercive fields. Long lengths of fibres are readily poled in this way.

To unequivocally establish that the internal copolymer layer was macroscopically poled we adopt a two-step approach. First, we show that the internal piezoelectric modulation indeed translates to a motion of the fibre's surface using a heterodyne optical vibrometer ${ }^{24-25}$ at $\mathrm{kHz}$ frequencies where fibre dimension is much smaller than the acoustic wavelength. Second, we proceed to an acoustic wave measurement at $\mathrm{MHz}$ frequencies where the fibre dimension exceeds the wavelength and the fibre geometry alters the acoustic wave front. The fibre sample is electrically driven by a sine wave with the amplitude of $10 \mathrm{~V}$. The vibrating fibre surface serves as an oscillating reflector and Doppler-shifts the frequency of the reflected light (Figure 2a), yielding frequency-modulation (FM) side bands spaced at the modulation frequency $\omega_{\mathrm{D}}$. The amplitudes of the side bands are proportional to the velocity amplitude of the vibration. Figure $2 \mathrm{~b}$ is the observed side bands from the cylindrical fibre of Figure 1, driven at frequencies from 1.3 to $1.9 \mathrm{kHz}$ thereby establishing a macroscopic piezoelectric response from the embedded ferroelectric layers. The side band amplitude modulation response is found at $\sim-60 \mathrm{~dB}$ below the main beat tone around these frequencies. Figure $2 \mathrm{c}$ shows the same frequency-modulation side bands for a rectangular fiber with an embedded planar piezoelectric layer, fabricated using a technique similar to the one described above (Supplementary Information). The rectangular geometry couples more efficiently to the optical beam leading to a marked improvement in the signal measured in the side bands compared to the immediate background. It also leads to a 20 $\mathrm{dB}$ increase in the side band amplitude with respect to the heterodyne sub-carrier. The ability to optimize the external fibre geometry with respect to the measurement system and application is another compelling property of this approach. 
The fibre draw is realized in a stress and temperature regime dominated by viscous forces (as opposed to surface tension) allowing for non-circular geometries to be realized. Controlling the precise shape of the fibre allows one in principle to tailor the acoustic wave front and its associated radiation pattern in the high-frequency limit where the acoustic wavelength is smaller than the lateral dimension of the fibre. Figure $2 \mathrm{~d}$ illustrates the result of finite-element calculation of the acoustic wave front emanating from three distinct fibre geometries. As shown the fibre structure and its associated acoustic wave front share the same symmetry elements.

We follow with direct acoustic measurements, using the fibres both as an acoustic sensor and as an acoustic actuator centred at $1 \mathrm{MHz}$. Such a frequency range is typical in ultrasound imaging applications. A schematic of the setup is shown in Figure 3a. A water-immersion ultrasonic transducer (Olympus Panametrics-NDT, 1.0 MHz-centered) is coupled to a fibre sample across a water tank to match the acoustic impedance. The fibre sample is attached to the water tank surface via immersion gel, with the piezoelectric layer facing towards the transducer. The rectangular geometry further improves the acoustic directionality. At $\mathrm{MHz}$ frequencies, capacitive electromagnetic coupling between the transducer circuit and the receiver charge amplifier can be significant even with careful shielding and grounding ${ }^{26}$. To separate the acoustic signals from the electromagnetic interference, we use a pulsed excitation and time-gate the received signals, exploiting the 5 orders of magnitude difference in the propagation speed between acoustic and electromagnetic pulses. The temporal traces of the amplified voltages under a pulsed excitation are measured with a carrier frequency at $600 \mathrm{kHz}$ and a $52 \mu$ s temporal envelope at a $6.5 \mathrm{kHz}$ repetition rate. The time delay of the received pulses is consistent with acoustic propagation in water at $1470 \pm 30 \mathrm{~m} / \mathrm{s}$ (Figure 3b). Frequency domain characterizations of the flat rectangular piezoelectric fibres are performed with a fixed transducer-to-fibre distance, 
with the pulsed excitation and the time-gated signal processing (Figure 3c). The measured piezoelectric response of the fibre, both as a sensor and an actuator, essentially follows the intrinsic frequency profile of the transducer. Although the frequency range is limited here by the bandwidth of the transducer, polymeric piezoelectric elements are in principle broadband and the piezoelectric fibres could operate at a far broader range of frequencies. For example, similar fibres were used to generate audible sound between $7 \mathrm{kHz}$ and $15 \mathrm{kHz}$ with a driving voltage of $5 \mathrm{~V}$.

The potential to modulate sophisticated optical devices is illustrated by constructing a fibre with a Fabry-Perot (FP) optical cavity structure ${ }^{1,3}$ layered on an embedded piezoelectric element. The fabrication process is shown in Figure 4a. SEM images show that the piezoelectric FP rectangular fibre (Figure $4 \mathrm{~b}$ ) is $800 \mu \mathrm{m}$ wide and exhibits well-maintained preform-to-fibre dimensional ratio and adhesion of the structures. Reflectivity of the piezoelectric FP fibre is characterized with a Fourier transform infrared (FTIR) microscope (Bruker Optics, Tensor/Hyperion 1000), revealing the reflectivity reaching 90\% around $1500 \mathrm{~nm}$ (Figure 4c, inset). The spectral dip associated with FP resonant mode is identified at $1550 \mathrm{~nm}$. We again employ heterodyne interferometry to characterize the fibre vibration produced by the embedded piezoelectric element. In Figure 4c, the piezoelectric FP fibre is electrically driven by a sine wave at frequencies stepped from 1.5 through $8.5 \mathrm{kHz}$ with the amplitude of $10 \mathrm{~V}$, while the probe beam is focused on the FP structure to take advantage of the enhanced reflection from the bulk structure (see supplementary information). The frequency dependence of the side band amplitude suggests signals enhanced or dampened by underlying acoustic resonances of the fibre sample. These piezoelectric FP fibres are mechanically robust and yet flexible; and can be 
assembled into a fabric for large area coverage as shown in Figure 4d. The manifested colour of the fabric is the reflection from the third order band of the Fabry-Perot optical structure embedded in the fibres.

In summary, the design, fabrication and characterization of extended length of fibres that are piezoelectric transducers is reported. An internal domain comprising a material which crystallizes in the ferroelectric phase is confined by a viscous conductive polymer. These are combined with metal electrodes to form a macroscopic preform, which is subsequently thermally drawn into many metres of fibres with spatially ordered and electrically contacted layers with non-centrosymmetric crystalline microstructure. Excellent uniformity allows for effective poling of the ferroelectric domain at fields exceeding $60 \mathrm{MV} / \mathrm{m}$. Piezoelectric response was established across a wide range of frequencies from $\mathrm{kHz}$ to $\mathrm{MHz}$ ranges using direct acoustic as well as heterodyne optical interferometry approach. By demonstrating monolithic processing of piezoelectric transducers and Fabry-Perot optical resonator in a single fibre, we present interesting opportunities in extending fibres from traditionally static components to dynamic systems that can be electrically modulated in a controlled fashion. This could enable the development of several unprecedented technological capabilities. For example, the unique fibre form-factor allows piezoelectric transducers to be woven into low-cost fabrics that would be capable of direct fabric-to-fabric communications and ranging over large surface areas ${ }^{27}$. In another example, the thermal drawing process allows for miniaturization of piezoelectric probes to dimensions of hundreds of microns and possibly even tens of microns, which is essential for enabling, accurate pressure and flow measurements in very small blood vessels (inter-cranial), in vivo endovascular imaging and acoustic microscopy inside acoustically-opaque organs ${ }^{28}$. Finally, 
uniform piezoelectric fibres have been prepared in tens of meters, ideal for distributed sensing ${ }^{12}$ : their sensitivity to stress and strain combined with their low profile make them ideal for constructing minimally-perturbative (sparse) sensor meshes for studying large-area distributions of the pressure and velocity fields in myriads of fluid flow applications including oceanic current monitoring ${ }^{11}$. In addition, the capability of fabricating acoustic fibres with arbitrary crosssections allows for integrating additional ferroelectric functionalities in a single-fibre device, such as energy conversion ${ }^{29-30}$ and other actively modulated devices ${ }^{29}$. 


\section{References and Notes}

1 Benoit, G., Hart, S. D., Temelkuran, B., Joannopoulos, J. D. \& Fink, Y. Static and dynamic properties of optical microcavities in photonic bandgap yarns. Adv. Mater. 15, 2053-2056, (2003).

2 Larsen, T. T., Bjarklev, A., Hermann, D. S. \& Broeng, J. Optical devices based on liquid crystal photonic bandgap fibres. Opt. Express 11, 2589-2596, (2003).

3 Benoit, G., Kuriki, K., Viens, J. F., Joannopoulos, J. D. \& Fink, Y. Dynamic all-optical tuning of transverse resonant cavity modes in photonic bandgap fibers. Opt. Lett. 30, 1620-1622, (2005).

4 Kerbage, C., Hale, A., Yablon, A., Windeler, R. S. \& Eggleton, B. J. Integrated all-fiber variable attenuator based on hybrid microstructure fiber. Appl. Phys. Lett. 79, 3191-3193, (2001).

5 Li, L., Wylangowski, G., Payne, D. N. \& Birch, R. D. Broad-band metal glass singlemode fiber polarizers. Electron. Lett. 22, 1020-1022, (1986).

6 Bergot, M. V. et al. Generation of permanent optically induced 2nd-order nonlinearities in optical fibers by poling. Opt. Lett. 13, 592-594, (1988).

7 Townsend, P. D., Poustie, A. J., Hardman, P. J. \& Blow, K. J. Measurement of the refractive-index modulation generated by electrostriction-induced acoustic waves in optical fibers. Opt. Lett. 21, 333-335, (1996).

8 Fokine, M. et al. Integrated fiber Mach-Zehnder interferometer for electro-optic switching. Opt. Lett. 27, 1643-1645, (2002).

9 Carpi, F. \& De Rossi, D. Electroactive polymer-based devices for e-textiles in biomedicine. Ieee Transactions on Information Technology in Biomedicine 9, 295-318, (2005).

10 Curie, J. \& Curie, P. Développement par compression de l'électricité polaire dans les cristaux hémièdres à faces inclinées. C. R. Acad. Sci. Paris 91, 294, (1880).

11 Arnau, A. Piezoelectric transducers and applications. (Springer, 2008).

12 Abouraddy, A. F. et al. Towards multimaterial multifunctional fibres that see, hear, sense and communicate. Nat. Mater. 6, 336-347, (2007).

13 Kawai, H. Piezoelectricity of poly (vinylidene fluoride). Jap. J. Appl. Phys. 8, 975-976, (1969). 
14 Lovinger, A. J. Ferroelectric polymers. Science 220, 1115-1121, (1983).

15 Lando, J. B., Olf, H. G. \& Peterlin, A. Nuclear magnetic resonance and X-ray determination of structure of poly(vinylidene fluoride). J. Polymer Sci. Part a 4, 941, (1966).

16 Matsushige, K., Nagata, K., Imada, S. \& Takemura, T. The ii-i crystal transformation of poly(vinylidene fluoride) under tensile and compressional stresses. Polymer 21, 13911397, (1980).

17 Hart, S. D. Multilayer composite photonic bandgap fibers, Massachusetts Institute of Technology, (2004).

18 Deng, D. S. et al. In-Fiber Semiconductor Filament Arrays. Nano Letters 8, 4265-4269, (2008).

19 Furukawa, T. Ferroelectric properties of vinylidene fluoride copolymers. Phase Trans. 18, 143, (1989).

20 Lando, J. B. \& Doll, W. W. The polymorphism of Poly(vinylidene fluoride). J. of Macromol. Sci.-Phys. B2, 205, (1968).

21 Yagi, T., Tatemoto, M. \& Sako, J. Transition behavior and dielectric-properties in trifluoroethylene and vinylidene fluoride co-polymers. Polymer Journal 12, 209-223, (1980).

22 Koga, K. \& Ohigashi, H. Piezoelectricity and related properties of vinylidene fluoride and trifluoroethylene copolymers. J. Appl. Phys. 59, 2142-2150, (1986).

23 Kimura, K. \& Ohigashi, H. Ferroelectric properties of poly(vinylidenefluoridetrifluoroethylene) copolymer thin films. Appl. Phys. Lett. 43, 834-836, (1983).

24 Eberhardt, F. J. \& Andrews, F. A. Laser Heterodyne System for Measurement and Analysis of Vibration. Journal of the Acoustical Society of America 48, 603-609, (1970).

25 Huber, R., Wojtkowski, M., Taira, K., Fujimoto, J. G. \& Hsu, K. Amplified, frequency swept lasers for frequency domain reflectometry and OCT imaging: design and scaling principles. Opt. Express 13, 3513-3528, (2005).

26 Morrison, R. Grounding and shielding : circuits and interference. (IEEE Press ; WileyInterscience, 2007).

27 Lurton, X. An introduction to underwater acoustics : principles and applications. (Springer, 2002). 
28 Pinet, E. Medical applications: Saving lives. Nat Photonics 2, 150-152, (2008).

29 Lines, M. E. \& Glass, A. M. Principles and applications of ferroelectrics and related materials. (Clarendon Press, 1977).

30 Neese, B. et al. Large electrocaloric effect in ferroelectric polymers near room temperature. Science 321, 821-823, (2008).

\section{Methods}

Acoustic transmission measurement: The rectangular fibre sample used in the measurement for Figure 3 has a lateral dimension of $3 \mathrm{~mm}$, approximately twice that of the acoustic wavelength at $1 \mathrm{MHz}$. In this context, a rectangular fibre would show stronger directionality than that of a circular fibre. A $30 \mathrm{~mm}$ long section of the fibre under test is coupled to the water tank through ultrasound gel. The transducer-to-fibre distance is around $97 \mathrm{~mm}$, approximately 70 acoustic wavelengths at $1 \mathrm{MHz}$.

Supplementary Information accompanies the paper on www.nature.com/nature.

Acknowledgements This work was supported by ARO, DARPA, ISN, and the MRSEC program of NSF. The authors acknowledge A. F. Abouraddy, G. Benoit, M. Spencer, J. Rigling, M. Thompson, and J. F. Viens for their critical help and for discussions; S. A. Speakman for assistance with the XRD measurements; and E. L. Thomas for guidance.

Author Contributions Y.F. and J.D.J. conceived the architecture of piezoelectric fibres. S.E., Z.W. and N.C. designed and fabricated fibre samples, performed acoustic and heterodyne optical measurements. Z.W. constructed the acoustic transmission setup. P.T.R. designed and constructed the heterodyne optical setup. S.E. measured the FP/piezoelectric fibres. Z.M.R. and A.M.S. performed thin-film deposition. D.S. performed SEM imaging. S.E., Z.W., N.C., F.S., J.D.J and Y.F. co-wrote the manuscript.

Author Information Reprints and permissions information is available at npg.nature.com/reprints . Correspondence and requests for materials should be addressed to Y.F. (yoel@mit.edu) 


\section{Figure Legends}

Figure 1 Structure of piezoelectric fibres. a, Schematic of the fabrication process of a cylindrical piezoelectric fibre. A preform is constructed by consolidating a shell of P(VDF-TrFE), shells containing CPC/indium electrodes, and PC cladding. b, SEM micrograph of the cross section of a cylindrical piezoelectric fibre. c, XRD patterns of $\mathrm{P}(\mathrm{VDF}-\mathrm{TrFE})$ samples extracted from drawn fibres and taken from melt-pressed films used in the preforms. The diffraction peaks indicate $\beta$-phase $\mathrm{P}(\mathrm{VDF}-\mathrm{TrFE})$.

Figure 2 Acoustic emission from piezoelectric fibres. a, Schematics of the laser Doppler vibrometer used to characterize the speed and frequency of the surface vibration of a fibre modulated at frequency $\omega_{D}$. b, c, Measured frequency-modulation side bands as a function of $\omega_{D}$ for piezoelectric fibres with cylindrical and rectangular cross sections respectively. The frequency range is illustrated in panel $b$ as a red box. $d$, Near-field pressure patterns of the acoustic emission at $1.3 \mathrm{MHz}$ from a circular fibre, a triangular fibre and a rectangular fibre with cross-sectional dimensions around $2 \mathrm{~mm}$.

Figure 3 Acoustic transmission characterization. a, Experimental setup for acoustic characterization of piezoelectric fibres. Acoustic wave travels across a water tank from a waterimmersion acoustic transducer to a fibre sample, and vice versa. b, Temporal traces of electrically-amplified acoustic signals detected by a piezoelectric fibre, shown together with the excitation signals. c, Acoustic signal detected (blue curve) and emitted (red curve) by a piezoelectric rectangular fibre around $1 \mathrm{MHz}$. The dotted line is the power spectrum of the (1 MHz-centred) transducer used. 
Figure 4. Integrated piezoelectric-modulated optical fibre. a, Schematic of the fabrication process of an integrated piezoelectric FP rectangular fibre. A FP optical cavity is embedded with the piezoelectric structure in a preform. The preform is thermally drawn into a micro-structured fibre. b, SEM micrograph of the cross section of an integrated piezoelectric FP fibre. c, Piezoelectricity of the fibre characterized with the fibre-optic heterodyne interferometer. Inset: Reflection spectrum of the piezoelectric FP fibre measured with FTIR. d, Two-dimensional device fabric constructed by knitting the piezoelectric/FP fibres as threads. Inset is a photograph of an individual fibre. 


\section{Supplementary Information:}

\section{Multimaterial Piezoelectric Fibres}

S. Egusa, ${ }^{1}$ Z. Wang, ${ }^{1,2}$ N. Chocat, ${ }^{3}$ Z. M. Ruff, ${ }^{3}$ A. M. Stolyarov, ${ }^{1,4}$ D. Shemuly, ${ }^{3}$ F. Sorin, ${ }^{1,3}$ P. T. Rakich, ${ }^{1,5}$ J. D. Joannopoulos, ${ }^{1,2}$ Y. Fink ${ }^{1,3^{*}}$

${ }^{1}$ Research Laboratory of Electronics, ${ }^{2}$ Department of Physics, and ${ }^{3}$ Department of Materials Science and Engineering, Massachusetts Institute of Technology, 77 Massachusetts Avenue, Cambridge, Massachusetts 02139

${ }^{4}$ School of Engineering and Applied Sciences, Harvard University, 29 Oxford Street, Cambridge, Massachusetts 02138

${ }^{5}$ Present Address: Sandia national Laboratories, P.O. Box 5800, Albuquerque, New Mexico 87185

* To whom correspondence should be addressed. E-mail: yoel@mit.edu 


\section{Preform fabrication of piezoelectric fibre}

Flat fibres were drawn from a macroscopic rectangular preform, $32 \mathrm{~mm}$ in width, $11 \mathrm{~mm}$ in thickness, and $25 \mathrm{~cm}$ long, which consisted of a $1.4 \mathrm{~mm}$-thick layer of P(VDF-TrFE) contacted by $\mathrm{CPC}$ and indium electrodes, and sandwiched between protective PC plates. The preform was consolidated in a hot press at $175^{\circ} \mathrm{C}$ and subsequently drawn in a three-zone vertical tube furnace with a top-zone temperature of $150^{\circ} \mathrm{C}$, a middle-zone temperature of $230^{\circ} \mathrm{C}$, and a down-feed speed of $1 \mathrm{~mm} /$ minute. The fibre dimensions were monitored with laser-micrometers. A capstan speed of 0.5-3 m/minute produces a fibre of width between about 2,000 and $600 \mu \mathrm{m}$ and a length of several hundred metres.
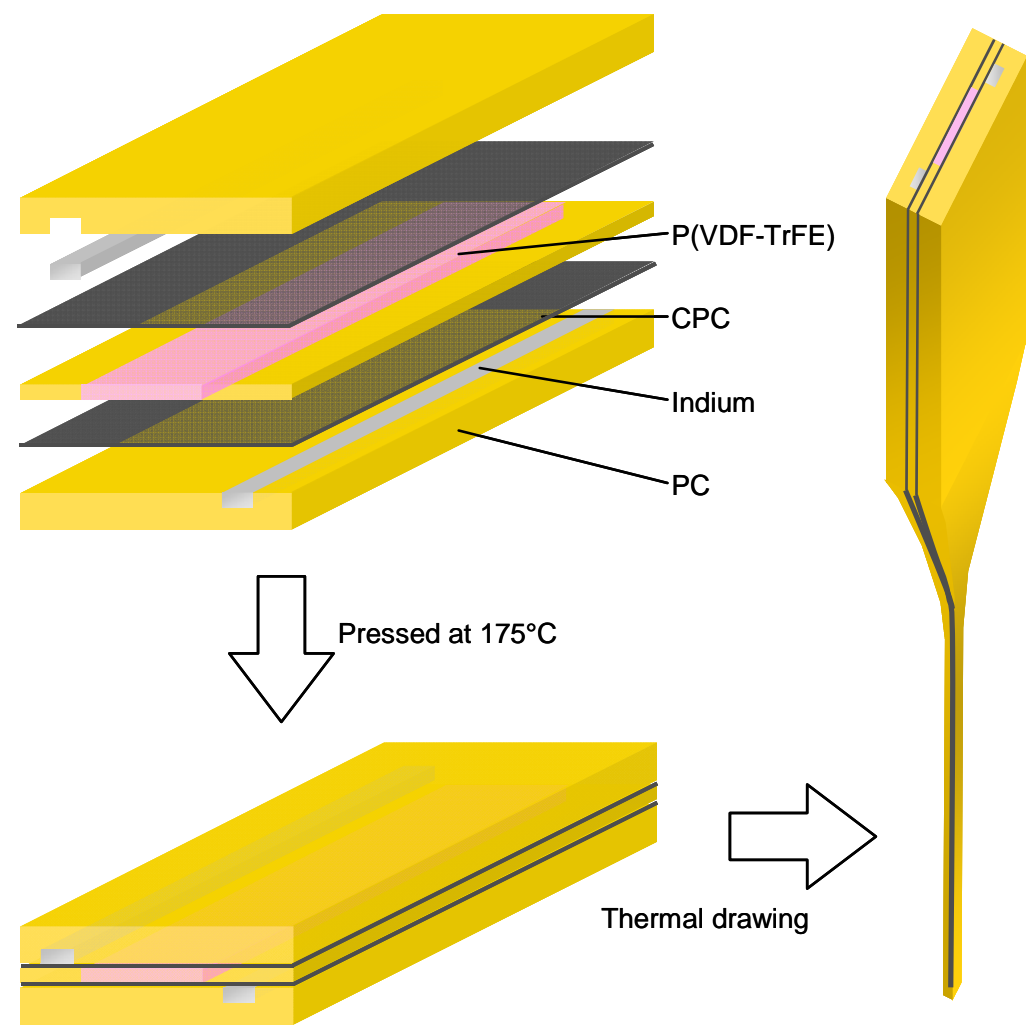
Figure S1 Schematic of the fabrication of rectangular fibres. A $1.4 \mathrm{~mm}$-thick layer of $\mathrm{P}(\mathrm{VDF}-\mathrm{TrFE})$ is sandwiched with $\mathrm{CPC}$ and indium electrodes and then thermally pressed at $175^{\circ} \mathrm{C}$ with PC plates on top and bottom.

\section{Heterodyne Laser Vibrometry}

The setup consists of a frequency-swept $(1530-1570 \mathrm{~nm}$, at $80 \mathrm{~nm} / \mathrm{s})$ laser (Agilent Technologies, 8164B) coupled to a fibre-optic Michelson interferometer (L2) and a Fabry-Perot frequency reference (L1). The two arms of the Michelson interferometer differ in length and generate a heterodyne beat tone. In one arm L2-S, light is focused onto vibrating surface of the fibre sample, where the Doppler shift of the reflected light produces additional frequencymodulation sidebands on the beat tone. The Fabry-Perot reference (L1) provides real-time frequency calibration. With this information, the frequency chirp in swept-laser source is compensated when processing the interferometry data. 


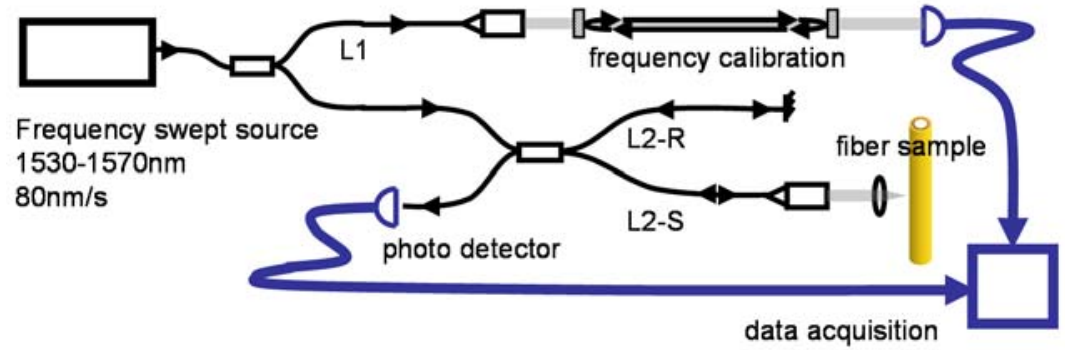

Figure S2 The optic setup of the heterodyne optical interferometer that serves as a laser vibrometer to probes the fibre surface.

\section{Fibre uniformity}

The stability of the draw is monitored by a continuous in-line measurement of the external dimensions of the fibre by a laser micrometer. The standard deviation is calculated over a $10 \mathrm{~cm}$ window in real-time and maintained at a level below $1 \%$ by controlling the draw stress. Since uniformity of the external geometry of the fibre does not ensure that of the internal structure, Scanning Electron Microscopy (SEM) is used to image the cross-sections of the fibre and evaluate the uniformity of the piezoelectric polymer layer thickness across its width. We find a standard deviation of $3 \%$ in the samples studied. To investigate the uniformity of the structure along the length of the fibre, we cut a one metre long portion of the fibre into $3 \mathrm{~cm}-1$ long segments, on which we measure the capacitance. Fluctuation in the averaged thickness of the piezoelectric $\mathrm{P}(\mathrm{VDF}-\mathrm{TrFE})$ layer should result in a proportional fluctuation in the capacitance. We find a standard deviation of $4 \%$. 
Figure 1.

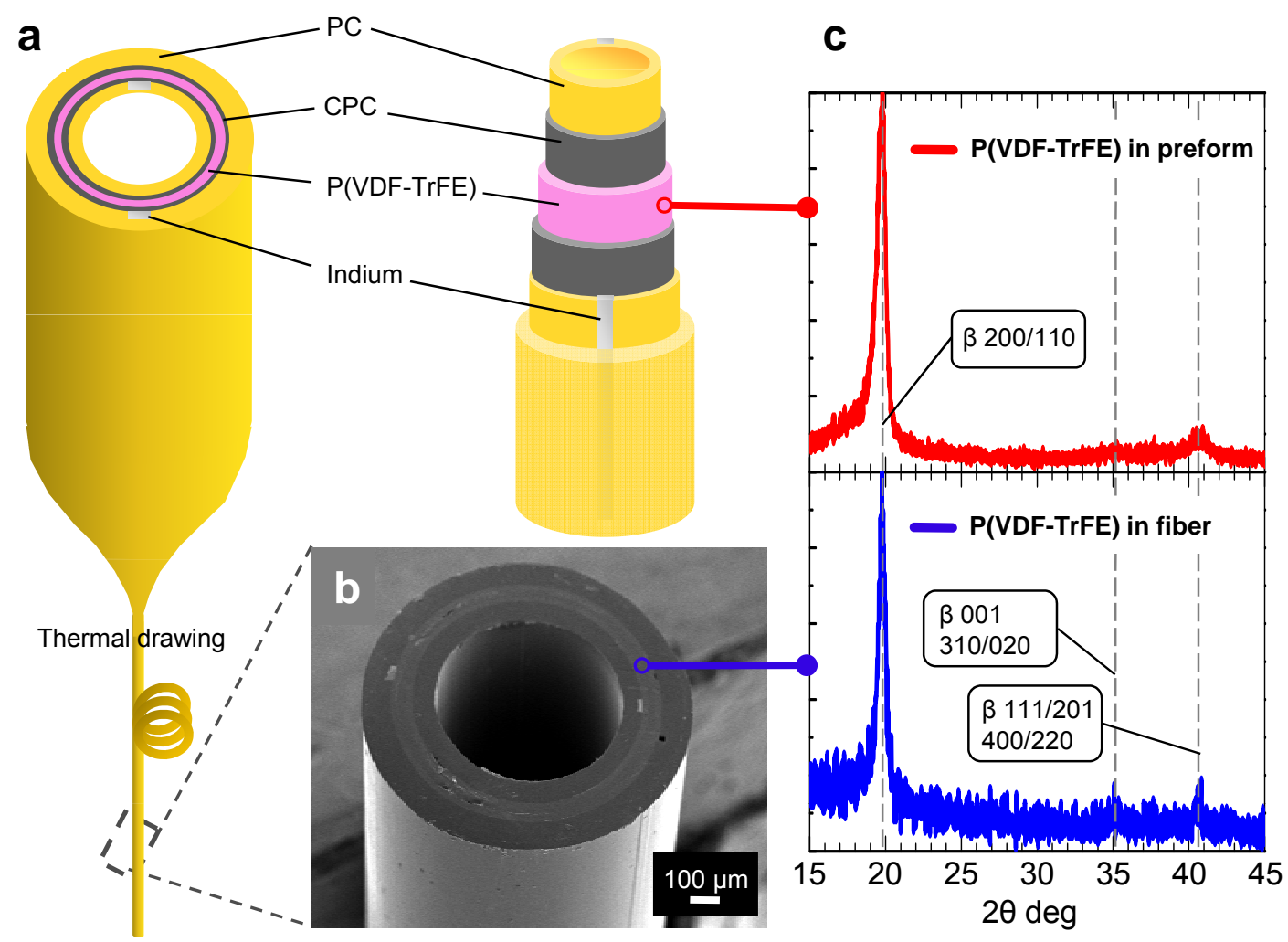

Piezoelectric Fibres Egusa et. al. 


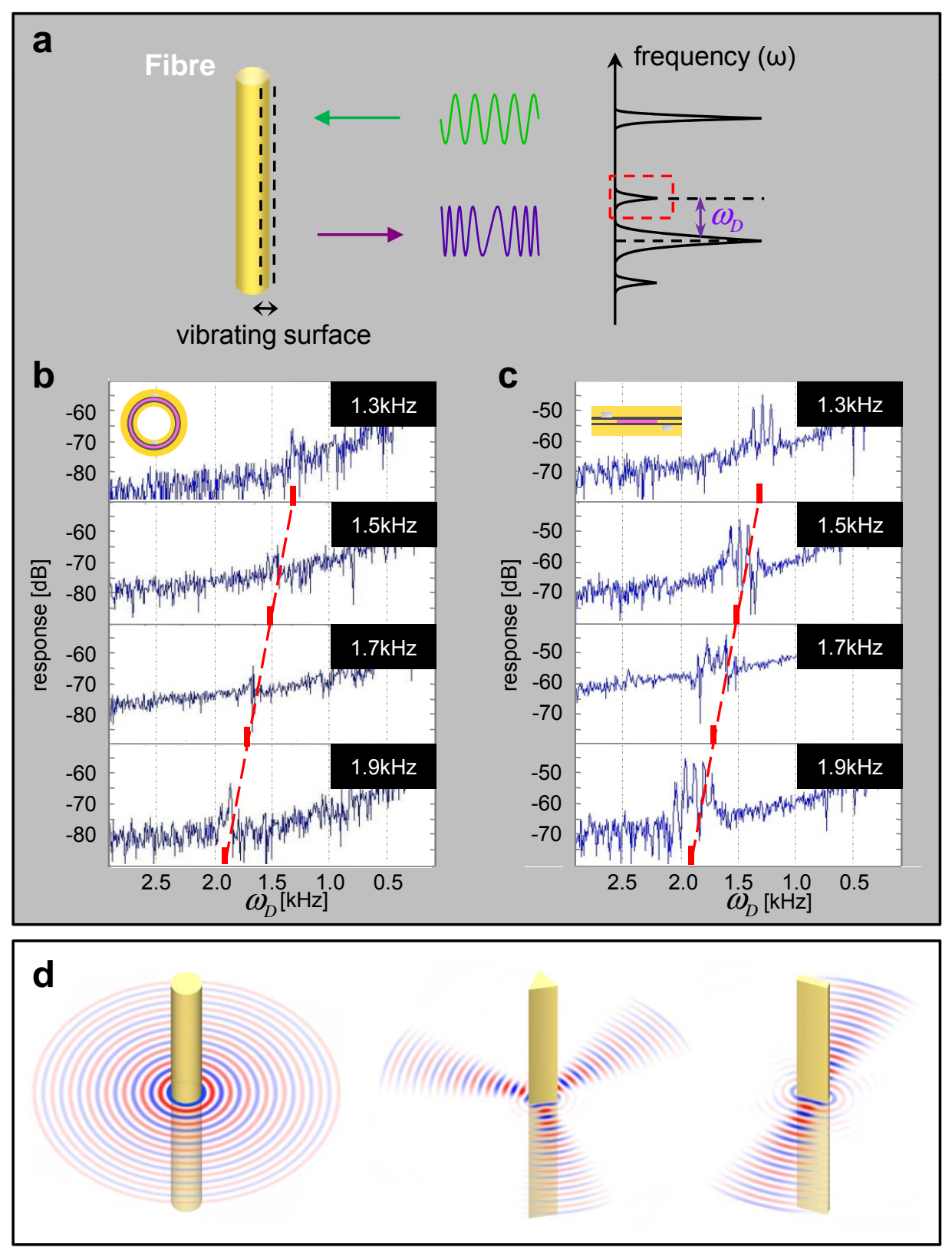

Figure 2.

Piezoelectric Fibres Egusa et. al. 


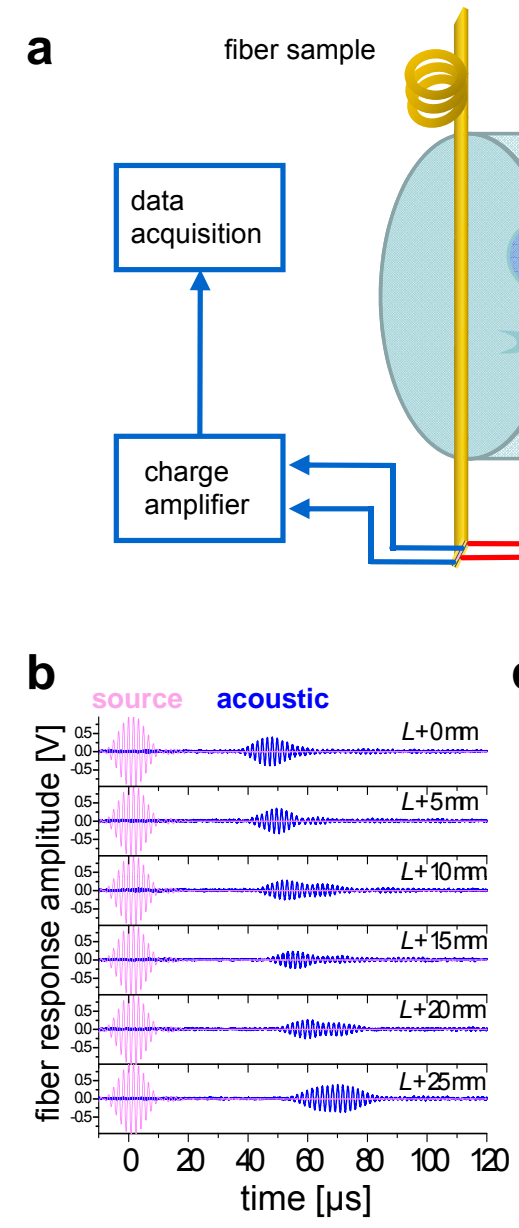

water tank

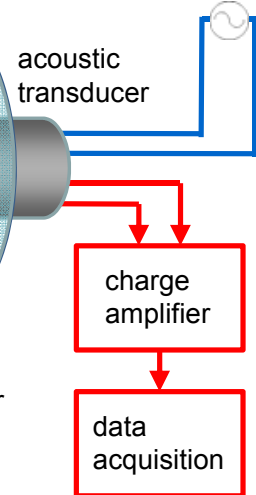

Piezoelectric Fibres Egusa et. al. 
Figure 4.

a
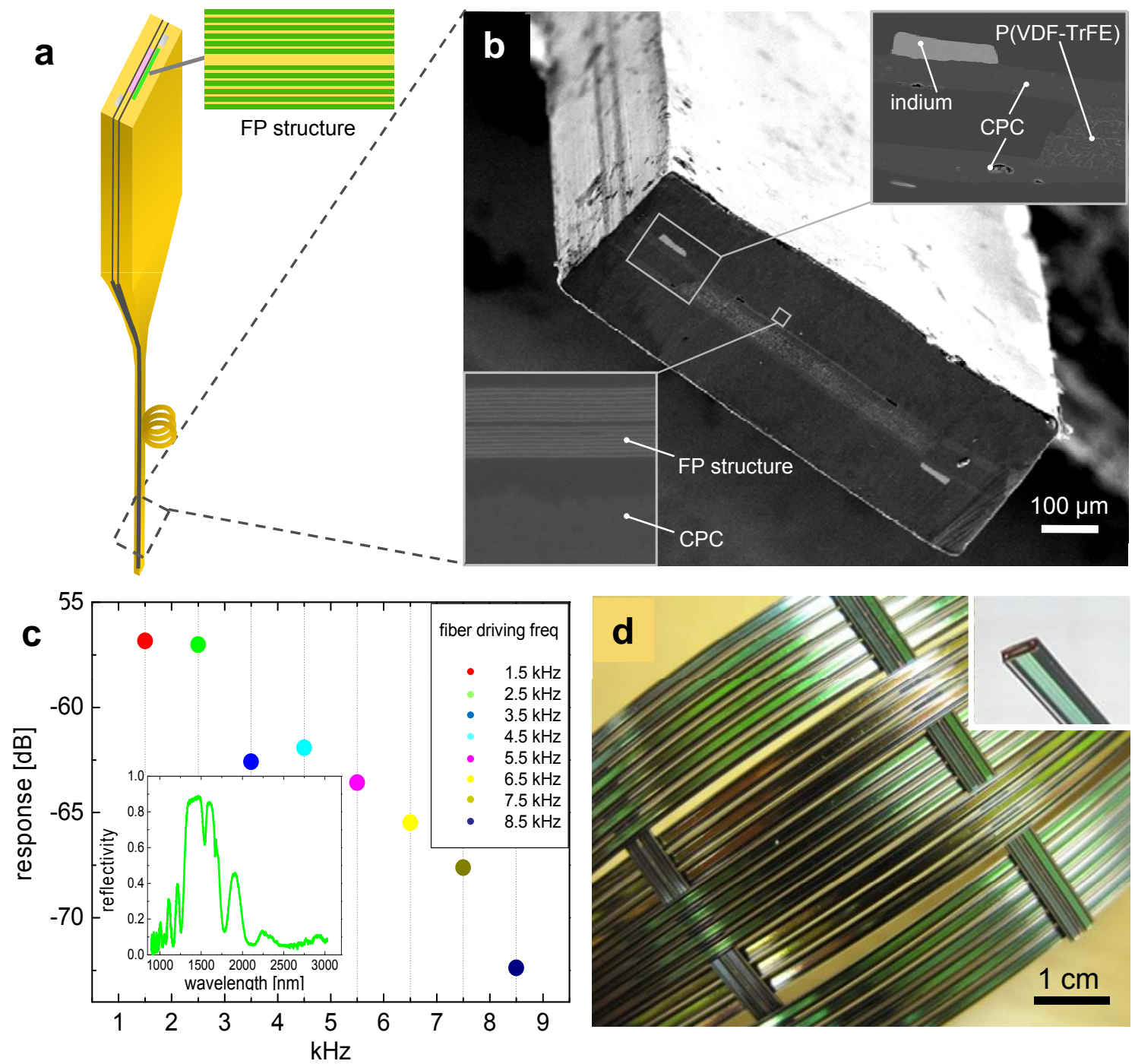

Piezoelectric Fibres Egusa et. al. 